\title{
Stand und Weiterentwicklung des QSR-Verfahrens
}

Das Verfahren „Qualitätssicherung mit Routinedaten“ (QSR) ist ein aufwandsarmes Qualitätsmessverfahren auf der Basis von anonymisierten AOK-Routinedaten, das sich an Ergebnisqualität orientiert. QSR wurde im Jahr 2002 als gemeinsames Entwicklungsprojekt des Wissenschaftlichen Instituts der AOK (WIdO), des AOK-Bundesverbands, der Helios Kliniken GmbH und des Forschungs- und Entwicklungsinstituts für das Sozial- und Gesundheitswesen Sachsen-Anhalt initiiert und wird seit 2008 vom WIdO kontinuierlich weiterentwickelt $[1,2]$. Der zentrale Vorteil von QSR gegenüber traditionellen Qualitätssicherungsverfahren besteht darin, dass auch Ereignisse im Therapieverlauf nach dem zu bewertenden Krankenhausaufenthalt in die Qualitätsmessung einfließen.

\section{Öffentliche Berichterstattung}

Im Jahr 2010 wurden die QSR-Ergebnisse zu ausgewählten Leistungsbereichen erstmals der interessierten Öffentlichkeit im AOK-Krankenhausnavigator im Rahmen eines Infoportals in Kooperation mit der Weissen Liste der Bertelsmann Stiftung zur Verfügung gestellt. Sie werden jährlich aktualisiert. Wesentliche Unterschiede zur Darstellung der Ergebnisse im QSR-Klinikbericht sind die namentliche Nennung der Kliniken und eine laienverständliche Aufbereitung der Daten. Dazu werden die QSR-Ergebnisse verdichtet und die Bewertung der Krankenhäuser kategorisiert.

\section{QSR-Klinikbericht}

Der QSR-Klinikbericht liefert detaillierte Informationen zur Ergebnisqualität für eine Klinik. Dabei ist ein Vergleich der Ergebnisqualität der jeweiligen Klinik hinsichtlich der einzelnen Indikatoren mit dem Bundesdurchschnitt möglich. Der Bericht enthält jahresbezogen eine Vielzahl aufbereiteter Kennzahlen wie z.B. Indikatoren zu ausgewählten Leistungsbereichen, die nach dem Zeitpunkt des Auftretens - im Startfall oder nach Klinikentlassung - unterschieden werden. Mit der Neugestaltung im Jahr 2011 umfasst der QSR-Klinikbericht auch die im AOK-Krankenhausnavigator veröffentlichten Mehrjahres-Ergebnisse.

\section{QSR-Indikatoren zur Cholezystektomie} Im Jahr 2011 wurden unter Experten-Einbindung QSR-Indikatoren für den Leistungsbereich Cholezystektomie entwickelt. Die Ergebnisse werden 2011 sowohl im AOK-Krankenhausnavigator als auch im QSR-Klinikbericht veröffentlicht.
Methodische Weiterentwicklung

Über die methodischen Grundlagen des QSR-Verfahrens informiert seit Juli 2011 eine neu eingerichtete Website (www.qualitaetssicherung-mitroutinedaten.de). Sie ist die zentrale Methodenreferenzstelle und richtet sich sowohl an die interessierte Fachöffentlichkeit und Patienten als auch an Kliniken.

Durch den neu gegründeten Wissenschaftlichen Beirat zum QSR-Verfahren sowie durch die Gründung eines Expertenpanels Kardiologie erfolgt eine verstärkte Einbeziehung von Experten-Expertise bei der Weiterentwicklung des QSR-Verfahrens. Weiterhin wurde die QSR-Methodik zu den bereits im AOK-Krankenhausnavigator veröffentlichten Leistungsbereichen (Endoprothetik) Fachgruppen vorgestellt und aktualisiert. Nach der Entwicklung von Qualitätsindizes [3], wurden insbesondere die Risikoadjustierungsverfahren verfeinert.

\section{Ausblick}

Schwerpunkte bei der Weiterentwicklung des QSR-Verfahrens sind insbesondere die Entwicklung neuer Indikatoren, die Einbeziehung sektorenübergreifender Ansätze, die Weiterentwicklung der Methodik zur Bildung von Qualitätsindizes und die Erschließung neuer statistischer Analyseverfahren. Derzeit werden QSR-Indikatoren für diagnostische und interventionelle Herzkatheter mit Unterstützung durch das Expertenpanel Kardiologie entwickelt, sodass die sektorenübergreifende Perspektive von QSR zunehmend mehr Raum erlangt. Auf der Produktseite werden die Informationssysteme für Patienten im Bereich der öffentlichen Berichterstattung und für die Krankenhäuser (QSR-Klinikbericht) weiter ausgebaut.

Autorenerklärung: Der Autor arbeitet für das WIdO und den AOK-Bundesverband am Projekt „Qualitätssicherung mit Routinedaten (QSR)“.

\section{Literatur}

1 AOK-Bundesverband, Forschungs- und Entwicklungsinstitut für das Sozial- und Gesundheitswesen Sachsen-Anhalt (FEISA), Helios Kliniken, Wissenschaftliches Institut der AOK (WIdO) (Hrsg). Qualitätssicherung der stationären Versorgung mit Routinedaten (QSR) - Abschlussbericht. Bonn: 2007; Available from: http://www.wido.de/fileadmin/wido/downloads/pdf_krankenhaus/wido_kra_qsrabschlussbericht_0407.pdf(7. März 2011)

2 Heller G. Zur Messung und Darstellung von medizinischer Ergebnisqualität mit administrativen Routinedaten in Deutschland. Bundesgesundheitsblatt - Gesundheitsforschung - Gesundheitsschutz 2008; 10: 1173-1182

3 Heller G. Qualitätssicherung mit Routinedaten - Aktueller Stand und Weiterentwicklung., In: Klauber J, Geraedts M, Friedrich J (Hrsg). Krankenhausreport 2010. Stuttgart: Schattauer: 2010: 239-253
J. Klauber

Qualitätsmanagement

Schlüsselwörter Qualitätssicherung

Ergebnisqualität

Routinedaten

Versorgungsforschung

Keywords

quality assurance

outcome quality

administrative data

health services research

Institut

Wissenschaftliches Institut der AOK (WIdO), Berlin

Bibliografie

DOI 10.1055/s-0031-1286096

Dtsch Med Wochenschr 2011;

136: S67 - (c) Georg Thieme

Verlag KG Stuttgart · New York . ISSN 0012-0472

Korrespondenz

Jürgen Klauber

Geschäftsführer

Wissenschaftliches Institut der AOK (WIdO)

Rosenthaler Straße 31

10178 Berlin

Tel. 030/34646-2137

eMail juergen.klauber@

wido.bv.aok.de 